\title{
A Detailed Study of the Properties of Smoke Particles Produced from both Flaming and Non-Flaming Combustion of Common Mine Combustibles
}

\author{
INOKA ERANDA PERERA, and CHARLES D. LITTON \\ US Department of Health \& Human Services, Centers for Disease Control \& Prevention \\ National Institute for Occupational Safety and Health, Pittsburgh Research Laboratory \\ 626 Cochrans Mill Road, PO Box 18070, Pittsburgh, PA 15236, USA
}

\begin{abstract}
This paper presents the results obtained from detailed studies of the properties of smoke particles produced from a wide range of flaming and non-flaming combustible materials and discusses how these properties impact early-warning fire detection as well as the hazards of smoke particle toxicity and reduced visibility that can significantly affect life safety. Data acquired include discrete angular scattering at wavelengths of $635 \mathrm{~nm}$ and $532 \mathrm{~nm}$; visible light obscuration; light extinction at a wavelength of $532 \mathrm{~nm}$ and total light scattering at a wavelength of $520 \mathrm{~nm}$; the responses of calibrated combination ionization/photoelectric smoke sensor; and total mass concentrations. These data are subsequently used to define the size, morphology and radiative transfer properties of the fractal aggregate smoke particles including radius of gyration, primary particle diameter, number of primary particles per aggregate, mass of an aggregate, mass extinction, scattering and absorption coefficients and the resultant albedo. Scanning electron microscope (SEM)/transmission electron microscope (TEM) data and computer-generated fractal aggregates are compared to determine similar morphologies and then used to calculate theoretical values of scattering, absorption, and extinction efficiencies using both the discrete dipole approximation (DDA) and the Rayleigh-Debye-Gans (RDG) approximation for subsequent comparison to the experimental data. These data and analyses indicate that significant differences exist between flaming and non-flaming smoke particles in terms of size, morphology and radiative transfer properties. From a practical viewpoint, the analyses also indicate possible techniques for development of improved early warning fire sensors and smarter, discriminating fire sensors that can function in hostile, contaminated atmospheres such as mines and tunnels. These atmospheres may contain significant levels of combustion products from internal combustion engines, such as diesels, that are used routinely in underground mines. In addition, the much higher albedos measured for non-flaming smoke particles are indicative of significantly lower carbon content and higher levels of volatile organic compounds that have the potential for increased acute toxicity due to their higher reactivity. The paper demonstrates how the basic data can be used to implement improved fire detection systems and improve our ability to assess hazards resulting from potentially catastrophic mine fires.
\end{abstract}

KEYWORDS: smoke, fractal aggregates, carbonaceous aerosol, detection.

\section{NOMENCLATURE LISTING}

$\begin{array}{llll}d_{g} & \text { number mean diameter }(\mathrm{cm}) & M_{a} & \text { mass of a fractal aggregate }(\mathrm{g}) \\ D_{f} & \text { fractal dimension } & m & \text { complex index of refraction, } m=n+i k_{e} \\ d_{p} & \text { diameter of primary particles }(\mathrm{nm}) & n_{p} & \text { number of primary particles per aggregate } \\ d_{10} & \text { count mean diameter }(\mathrm{cm}) & N & \text { number of aggregate per } \mathrm{cm}^{3} \\ D_{i} & \text { ion diffusion coefficient }\left(\mathrm{cm}^{2} / \mathrm{s}\right) & R_{g} & \text { radius of gyration }(\mathrm{nm}) \\ E_{m} & \left(m^{2}-1\right) /\left(m^{2}+2\right)=\operatorname{Im}\left[\left(m^{2}-1\right) /\left(m^{2}+2\right)\right] & t & \text { time }(\mathrm{s}) \\ F_{m} & \left(m^{2}-1\right) /\left(m^{2}+2\right)=\left|\left(m^{2}-1\right) /\left(m^{2}+2\right)\right|^{2} & x_{p} & \text { size parameter, } \pi d_{p} / \lambda \\ I_{0} & \text { incident intensity of the light }(\text { arb units) } & \sigma_{e x t} & \text { mass specific extinction }\left(\mathrm{m}^{2} / \mathrm{g}\right) \\ I & \text { reduced intensity of light }(\operatorname{arb} \text { units }) & \sigma_{\text {sca }} & \text { mass specific scattering }\left(\mathrm{m}^{2} / \mathrm{g}\right) \\ k_{f} & \text { fractal pre-factor } & \sigma_{a b s} & \text { mass specific absorption }\left(\mathrm{m}^{2} / \mathrm{g}\right) \\ k & \text { wave vector, } 2 \pi / \lambda,\left(\mathrm{nm}^{-1}\right) & \lambda & \text { wavelength }(\mathrm{nm}) \\ l & \text { path length }(\mathrm{m}) & \rho_{p} & \text { particle density }\left(\mathrm{g} / \mathrm{m}^{3}\right) \\ M & \text { aerosol mass concentration }\left(\mathrm{mg} / \mathrm{m}^{3}\right) & \theta & \text { scattering angle }(\text { degrees })\end{array}$




\section{INTRODUCTION}

Most fires produce significant levels of smoke particles, or combustion aerosols that can generally be described as fractal or fractal-like aggregates (FA) where each fractal aggregate contains many small, primary particles connected together in a random fashion. Fractal aggregates are generally characterized by a set of parameters such as the fractal, or Hausdorff, dimension, $D_{f}$, the radius of gyration, $R_{g}$, the diameter or radius of each primary particle forming the aggregate, and the average number of these primary particles per aggregate [1-6] . The fractal dimension is generally a measure of the shape of the aggregate, where values in the range of 1.6 to 1.9 correspond to aggregates that have an elongated, chain-like structure with very little overlap of individually connected particles. Larger fractal dimensions, generally in the range of 2.1 to 2.3 , correspond to aggregates that are more compact, with individual particles significantly overlapped or 'necked' together. Previous studies of flame soot indicate that soot particles from fuel-lean and stoichiometric combustion generally exhibit the former aggregate structure. As combustion becomes more fuel-rich, the aggregate structure gradually evolves to the more compact form, with fractal dimensions increasing with the fuel-air ratio [6-9]. While extensive studies of the structure of flame soot exist in the literature, similar studies of the post-flame aerosols, i.e., smoke particles expelled into the atmosphere, are sparse. Although it is generally accepted that smoke particles produced from wellventilated flaming fires produce FAs with lower $D_{f}$ values while those from non-flaming fires have much higher $D_{f}$ values, the quantitative data are lacking.

In order to more completely describe the fractal aggregates produced from typical fires, this paper details the results of experiments conducted to quantify the optical scattering, extinction and absorption properties of fractal aggregates produced from different combustion sources and different modes of combustion-i.e., flaming and non-flaming - that are important to fire safety as well as to the more general area of carbonaceous atmospheric aerosols and their impact on climate and health. Measurements of mass scattering, mass extinction and, by difference, mass absorption coefficients are compared to data previously reported for carbonaceous aerosols from flaming and non-flaming fires. Discrete angular scattering data at four forward and two backward angles at two wavelengths of $532 \mathrm{~nm}$ and $635 \mathrm{~nm}$ are used to derive radii of gyration and fractal dimensions. In addition, the discrete angular scattering data and mass scattering, extinction and absorption coefficients are compared to detailed numerical computations using the discrete dipole approximation (DDA) and to approximate computations using the Rayleigh-Debye-Gans (RDG) approximation [10-15]. For these comparisons, it was found that the DDA and RDG solutions are in reasonable agreement with the data when the index of refraction is chosen appropriately, and that the size and shape of the fractal aggregates are also important but have less of an impact. The responses of a prototype instrument that combines an ionization chamber with discrete forward angular scattering are used to derive the primary particle diameters, number concentration of FAs, average mass of the fractal aggregate, and the average number of primary particles per aggregate. In addition, these prototype responses were used to assess and compare ionization-type and photoelectric-type smoke sensor sensitivities to aerosols from flaming and non-flaming combustion.

While presenting novel and unique data, the paper also discusses the potential relevance and applicability of the data in a number of diverse areas. In the areas of fire safety, these quantitative data could be used to improve the performance of smoke detectors by reducing false/nuisance alarms and discriminating between different combustion sources and different modes of combustion. The data also provide a possible means to improve the capability to predict the optical properties of smoke such as absorption, scattering and extinction coefficient - parameters that are needed to assess levels of visibility in escape routes and also to properly design emergency lighting systems to aid in evacuation, especially in underground mines and tunnels. The data may also be useful for the design and development of improved devices for monitoring and measurement/characterization of atmospheric aerosols produced from typical combustion processes, such as coal-fired power plants or metallurgical processing facilities, forest fires, and other combustion phenomena. Quantification of the properties of these fractal aggregates is also important for refining aerosol radiative transfer models, which are used to predict the effects of atmospheric aerosols on our changing climate where the chemistry, morphology, and carbon content of combustion-generated aerosols is of great significance. 


\section{EXPERIMENTAL}

For the studies described here, experiments were conducted using a standard Underwriters Laboratory, Inc. (UL 268) smoke chamber [16] connected to a combustion chamber containing a circular disk heater used to heat the solid combustibles. The combustible materials used in both flaming and non-flaming experiments were wood chips from dried Douglas-fir, Pittsburgh (Pgh) seam coal $(-9.4$ to $+6.7 \mathrm{~mm}$ mesh), styrene butadiene rubber (SBR) from a typical non-fire resistant conveyor belt, and small strips of polypropylene (a typical battery covering) cut from a $0.61 \mathrm{~m}$ square sheet $0.0381 \mathrm{~m}$ thick. Flaming experiments were also conducted using a small circular pool of No. 2 diesel fuel. The aerosols from the various combustion sources were produced within the combustion chamber and then flowed into the larger smoke chamber through a four-inch diameter duct. Once in the smoke chamber, the aerosol was mixed uniformly using two small circulating fans. Inside the smoke chamber, optical density of the aerosol was measured over a $1.48 \mathrm{~m}$ optical path length using an incandescent lamp and a standard photocell with a spectral response matching the spectral response of the human eye. In some of the earlier experiments, light extinction over a $0.65 \mathrm{~m}$ path at a wavelength of $532 \mathrm{~nm}$ was also measured within the smoke chamber using a small laser and silicon photodiode. In later experiments, the $532 \mathrm{~nm}$ laser and photodiode were connected at either end of a $0.6858 \mathrm{~m}$ long sealed steel pipe external to the smoke chamber and light extinction was measured by flowing the aerosol continuously from the smoke chamber through the pipe. During the experiments, the aerosols were continuously extracted from the smoke chamber using metal tubes inserted into the top of the smoke box and flowed to various measuring devices. In addition to the measurements of visible light obscuration and light extinction at $532 \mathrm{~nm}$, data acquired during the experiments also included discrete angular scattering at wavelengths of $635 \mathrm{~nm}$ and $532 \mathrm{~nm}$ and at four forward and two backward angles, $15^{\circ}$, $22 \frac{1}{1} 2^{\circ}, 30^{\circ}, 45^{\circ}, 135^{\circ}$ and $150^{\circ}$ using a precisely machined scattering chamber described in detail elsewhere [17]. The normalized angular scattering data were subsequently used to derive the fractal properties $R_{g}$ and $D_{f}$. In addition, continuous measurements of the aerosol mass concentrations were made using a calibrated TSI DustTrak 8520 or 8530 that extracted samples via one of the metal tubes connected to the smoke chamber.

Samples were also flowed from the smoke chamber to an Ecotech Aurora-1000 single wavelength integrating nephelometer for measurement of the total scattering efficiency at a wavelength of $520 \mathrm{~nm}$, and to a prototype sensor consisting of a well-defined ionization chamber and an optical scattering chamber for discrete measurements of angular scattering at $15^{\circ}$ and $30^{\circ}[18,19]$. Measurement of the mass concentrations were subsequently used to determine the discrete angular scattering sensitivities for the sixangle scattering module (intensity per unit mass concentration); the mass extinction coefficients for the broadband visible light obscuration and $532 \mathrm{~nm}$ light extinction; mass scattering coefficients at a wavelength of $520 \mathrm{~nm}$ from the nephelometer data; and ionization chamber and angular sensitivities from the prototype sensor in units of voltage change per unit mass concentration. Angular intensity data from the prototype sensor were also used to derive the radius of gyration for comparison to the results from the sixangle scattering module, and the ionization chamber response was used to determine the average number concentration of aggregate particles, average mass of an aggregate, primary particle diameter, and number of primary particles per aggregate. For all of the combustion sources, filter samples were taken for subsequent analysis using scanning electron microscopy (SEM) and transmission electron microscopy (TEM). For SEM analysis, smoke samples were collected onto $25 \mathrm{~mm}$ polycarbonate membrane filters (Millipore) topped with formvar-coated TEM grids using a $1.7 \mathrm{~L} / \mathrm{min}$ pump. TEM grids were pasted onto the SEM sample filters using double sided tape. These polycarbonate membrane filters were coated with gold/palladium prior to the SEM imaging and the formvar-coated TEM grids were directly placed in the sample chamber for the TEM imaging. SEM images were obtained using a JEOL 6400 scanning electron microscope (JEOL, Inc., Tokyo, Japan) and TEM images were viewed using a JEOL 1220 transmission electron microscope (JEOL, Inc.).

\section{THEORY}

The chemistry, morphology and size of fractal aggregates produced from combustion aerosols vary depending on the source and the nature of the fire from which they are generated, and these properties, in turn, produce unique scattering and extinction properties. Sorensen and others have used light scattering signatures (intensity versus angle) to derive average size (radius of gyration, $R_{g}$ ) and fractal dimension, $D_{f}$, through the use of $q$, the modulus of the scattering vector, defined in Eq. 1 [20-22]. 
$q=\frac{4 \pi}{\lambda} \sin (\theta / 2)$

where $\theta$ is the scattering angle measured from the forward direction and $\lambda$ is the wavelength, either 635 or $532 \mathrm{~nm}$ for the work reported here. For small angles, and with $q R_{g}<1$, scattering is in the so-called Guinier-Regime where the intensity is independent of the index of refraction and given approximately by the Guinier equation, as in Eq. 2.

$$
I(q) \approx 1-(1 / 3)\left(q R_{g}\right)^{2}
$$

Similarly, for large values of $q$ and $q R_{g} \gg 1$, the angular intensity varies according to Eq. 3 .

$$
I(q) \approx q^{-D_{f}}
$$

Thus a plot of $\ln (\mathrm{I}(q))$ versus $\ln (q)$ yields the fractal dimension, $D_{f}$. For the aggregate particles, the applicable fractal power law is given by Eq. 4 .

$n_{p}=k_{f}\left(R_{g} / d_{p}\right)^{D_{f}}$

Since $R_{g}$ and $D_{f}$ can be obtained from the angular scattering data, the primary particle diameter is the remaining parameter to be determined. Generally, SEM or TEM measurements provide sufficient information to determine $d_{p}$, but these techniques are unable to provide continuous, real-time information. To determine approximate values of $d_{p}$, the response of the ionization chamber from the prototype sensor is used in the following manner. For voltage changes less than about 1 to $1.2 \mathrm{~V}$, the change in voltage from the ionization chamber can be expressed as in Eq. 5 [18,23].

$\triangle C E V=(0.075) 2 \pi D_{i} d_{10} N$

The mass concentration, $M$, in $\mathrm{mg} / \mathrm{m}^{3}$, can be expressed by Eq. 6 .

$$
M=1 \times 10^{9}(\pi / 6)\left(d_{10}\right)^{3} N
$$

The ratio CEV/M, for an aggregate size distribution that is assumed to be lognormal with a geometric standard deviation of $\sigma_{g}=1.70$, is given by Eq. 7 .

$C E V / M=1.014 \times 10^{-9} D_{i} / d_{g}^{2}$

In a previous study, $D_{i}$ was found to have a value of $0.1522 \mathrm{~cm}^{2} / \mathrm{s}$, although the generally accepted value is much lower at $0.042 \mathrm{~cm}^{2} / \mathrm{s}$. Assuming the former value, Eq. 7 can be rearranged to yield $d_{g}$.

$d_{g}=1.242 \times 10^{-5} /(C E V / M)^{1 / 2}$

Assuming the same lognormal distribution, $\mathrm{d}_{10}$ in Eq. 5 is roughly 1.15 times $d_{g}$, and using the value of $D_{i}=$ $0.1522 \mathrm{~cm}^{2} / \mathrm{s}$, Eq. 5 can be rearranged to yield an expression for $N$, as in Eq. 9 .

$$
N=12.11\left(C E V / d_{g}\right)
$$


Since the aerosol mass concentration is the average mass of an aggregate, $M_{a}$, times the number concentration, $N, M_{a}$ is given by the simple Eq. 10 .

$M_{a}=\left(1 \times 10^{-9}\right)(M / N)$

Similarly, the mass of an aggregate is the mass of an individual primary particle (particle density, $\rho_{p}, \times$ particle volume, $\pi d_{p}{ }^{3} / 6$ ) times the average number of primary particles per aggregate. Using Eq. 4 , the average mass of an aggregate can then be expressed as in Eq. 11.

$M_{a}=k_{f}\left(R_{g} / d_{p}\right)^{D_{f}} \rho_{p} \pi d_{p}^{3} / 6$

And solving this expression for $d_{p}$ yields Eq. 12.

$d_{p}=\left[(6 / \pi)\left(M_{a} /\left(k_{f} \rho_{p} R_{g}^{D_{f}}\right)^{1 /\left(3-D_{f}\right)}\right.\right.$

Once $d_{p}$ is determined, the average number of primary particles per aggregate can then be determined. It should be noted that Eq. 10 implicitly assumes that the primary particles forming the aggregate just barely touch and that no overlap occurs. In general, this is approximately true for FAs with $D_{f}$ in the range of 1.7 to 1.9, but for higher fractal dimensions, significant overlap does occur, and this should be accounted for in the above expressions. Sorensen has derived simulations that relate the degree of overlap not only to $D_{f}$ but also to the fractal law pre-factor, $k_{f}$. In this study, the effects of overlap were also estimated for a fractal aggregate containing 74 primary particles using standard AutoCAD techniques [24]. Equating our overlap parameter to that used by Sorensen, it was found that for $D_{f}<1.8$, Eq. 10 is valid to within about $10 \%$. For a $D_{f}$ in the range of 2.0 to 2.3, Eq. 10 can overestimate the average aggregate mass by $50 \%$ to $70 \%$ [25]. In the data reported below, Eq. 10 is used directly without adjustment for overlap in the interest of brevity, although future analyses will include this effect.

In addition to the acquisition of discrete angular scattering data and the responses of the prototype sensor, light extinction measurements, as discussed above, were also made. For these data, the standard equation for light transmission is used, $I / I_{0}=\exp \left(-1 \times 10^{-3} \sigma_{e x t} M l\right)$. Light scattering measurements are made using the nephelometer, and the measured scattering coefficients divided by the mass concentrations yield the mass scattering coefficients, $\sigma_{s c a}$, directly. The mass absorp-tion coefficients, $\sigma_{a b s}$, are then obtained by difference, $\sigma_{a b s}=\sigma_{e x t}-\sigma_{s c a}$. In addition to the equations presented above, detailed numerical calculations were made using DDSCAT, a discrete dipole approximation algorithm maintained by Draine [11, 14]. Computations were performed for a fractal aggregate with varying degrees of overlap of the primary particles, a range of values of the index of refraction typical of combustion-generated aerosols, and at aggregate dimensions typical of those measured in this study. The values of fractal properties and index of refraction necessary in order for the detailed numerical calculations to agree with the data are also presented in the results section, not only for the characteristic angular scattering patterns but also for the albedos measured for the different combustion sources and two combustion modes.

In addition to the DDSCAT calculations, the Rayleigh-Debye-Gans approximation (RDG) can also be used to estimate angular scattering intensity distributions and the mass extinction, mass scattering and mass absorption coefficients. The expressions used to estimate the RDG angular distribution are described in detail in the literature [21,24]. For fractal aggregates, the mass absorption and mass scattering crosssections, $\sigma_{a b s}$ and $\sigma_{s c a}$, are given by the expressions from Dobbins et al. [1,26], in Eqs. 13 and 14.

$\sigma_{a b s}=6 \pi E_{m} / \lambda \rho_{p}$

$\sigma_{s c a}=\left(4 \pi n_{2} \chi_{p}^{3} F_{m} / \lambda \rho_{p} n_{1}\right)\left[1+\left(4 / 3 D_{f}\right)\left(k^{2} R_{g}^{2}\right)\right]^{-D_{f} / 2}$ 
where $\rho_{p}$ is the density of a primary particle, taken to be $1.86 \mathrm{~g} / \mathrm{cm}^{3}$, and

$n_{1}$ and $n_{2}$ are the first and second moments of the aggregate size distribution

In the case of large aggregates, $n_{2} \rightarrow\left(n_{p}\right)^{2}$ and $n_{1} \rightarrow n_{p}$ and with $k^{2}\left(R_{g}\right)^{2} » 1$, Eq. 16 reduces to Eq. 15 .

$\sigma_{s c a}=\left(4 \pi \chi_{p}^{3} k_{f} F_{m} / \lambda \rho_{p}\right)\left(3 D_{f} / 16 \chi_{p}^{2}\right)^{D_{f} / 2}$

These equations, along with the angular intensity equations, are used in the experimental section to estimate the angular intensity distributions and the mass scattering, mass extinction and mass absorption coefficients using the experimentally determined parameters appearing in Eqs. 15 and 17. This is done in order to assess the utility of the RDG approximation as a simpler method (compared to the detailed DDA or other numerical techniques) for estimating the radiative transfer properties for combustion-generated fractal aggregates.

\section{RESULTS AND DISCUSSION}

\section{Optical and SEM/TEM Data for Fractal Aggregate Smoke Particles}

Figure 1 displays the aerosol angular scattering signatures generated for both flaming and non-flaming fires for the commonly used combustibles, namely Douglas-fir wood, Pittsburgh seam coal, SBR rubber, polypropylene and No. 2 diesel fuel. The data indicate that each of the different aerosols have characteristic angular intensity distributions for both flaming and non-flaming modes. While it is beyond the scope of this present study to provide any greater detail, it is expected that these distributions reflect minor differences in the absorbing nature of the individual aerosols (i.e., their chemical composition) as well as small differences in the fractal aggregate structure and size. It is also evident that at forward angles the aerosols generated from non-flaming fires scatter with higher intensities than the aerosols from flaming fires. In fact, for all the forward angles and two wavelengths measured in these studies, the scattering intensity from nonflaming aerosols was $36 \%$ higher than those from flaming fires.
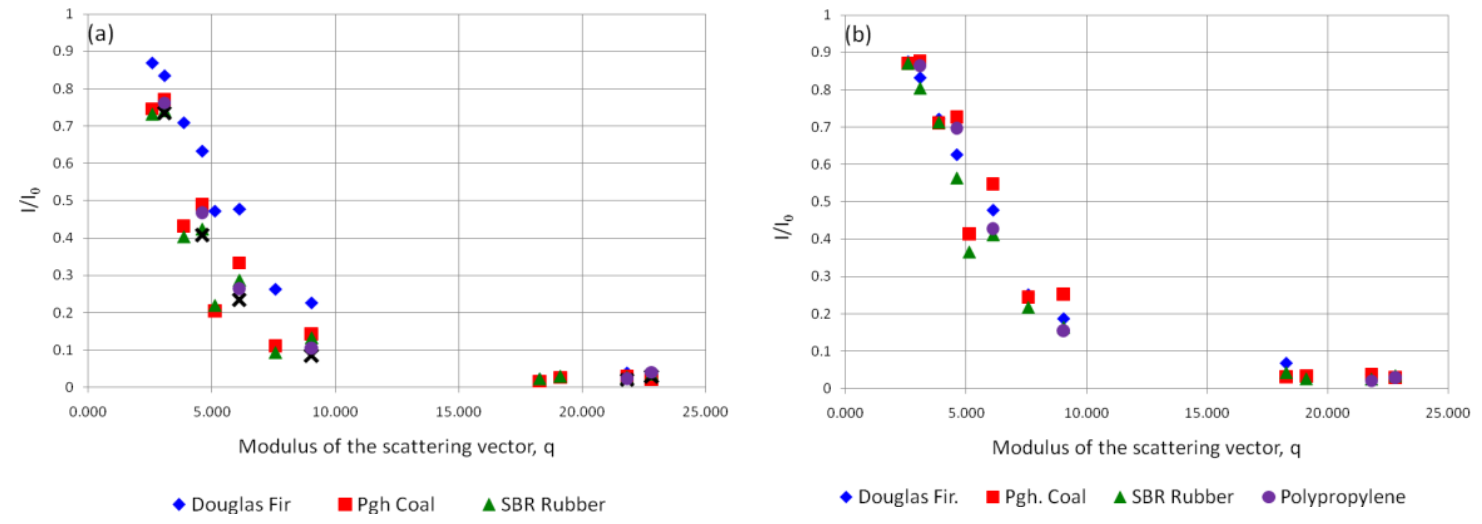

Fig. 1. Scattering intensities $\left(I / I_{0}\right)$ for aerosols produced from both (a) flaming and (b) non-flaming fires vs. modulus of the scattering vector, $q$, for different combustion sources.

The higher intensity for aerosols from non-flaming combustion is particularly evident in Fig. 2 where the average for each mode of combustion is presented. Higher scattering intensities for non-flaming fractal aggregates correlate with a greater degree of clumping or coagulation. Both the SEM images below (Fig. 4) and higher fractal dimensions (as discussed further in the manuscript) are consistent with these results. Increased scattering by aerosols from non-flaming fires is also consistent with greater sensitivity to these types of fires by photoelectric smoke sensors. 


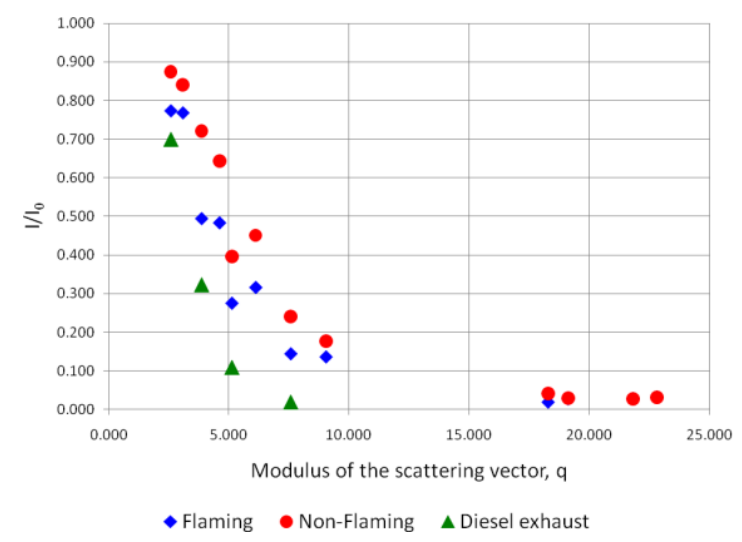

Fig. 2. Scattering intensities $\left(I / I_{0}\right)$ for flaming/non-flaming fires vs. modulus of the scattering vector, $q$.
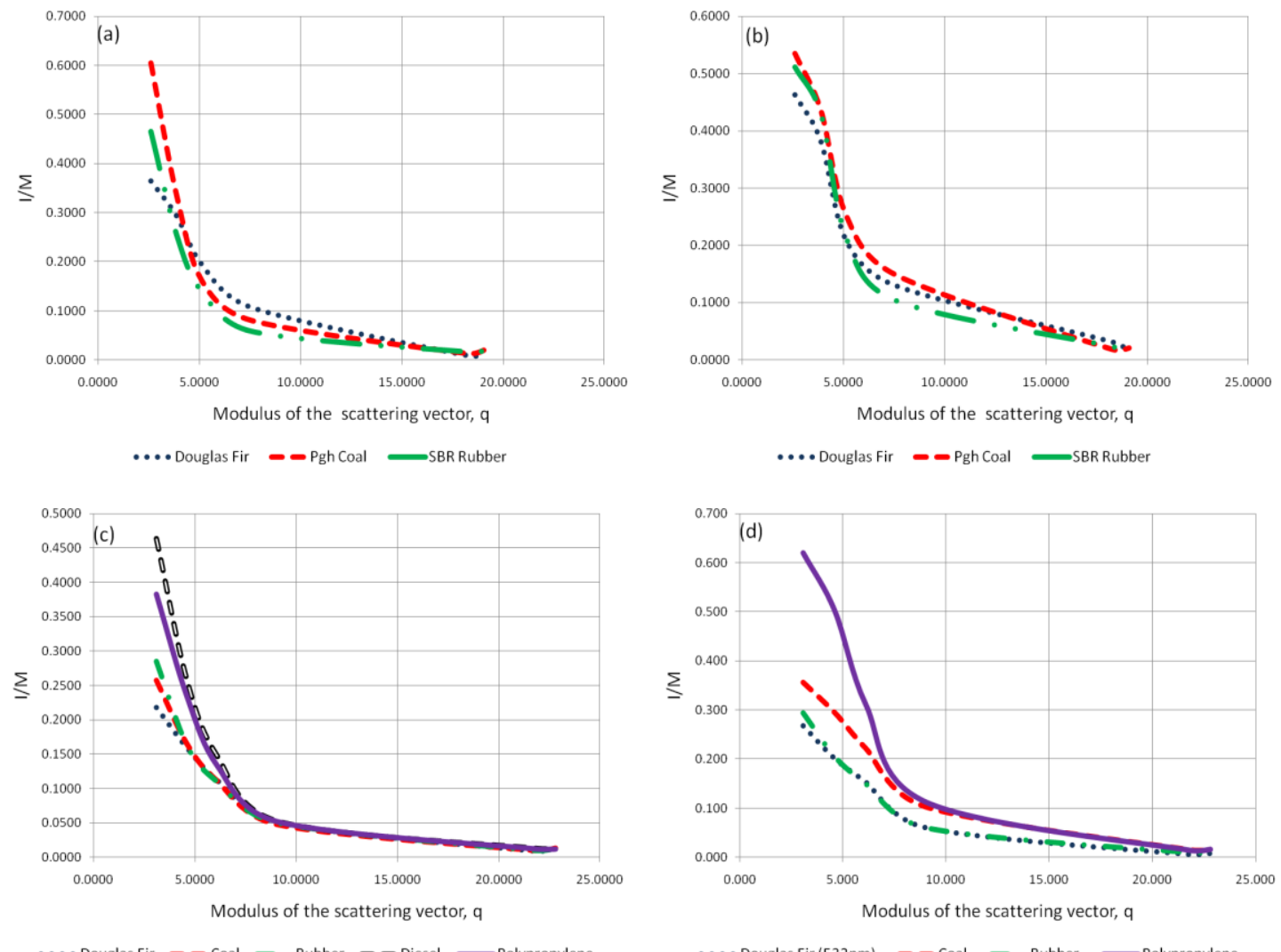

-..Douglas Fir - - Coal — Rubber o ס Diesel —Polypropylene

...Douglas Fir $(532 \mathrm{~nm}) \quad$ - Coal - Rubber Polypropylene

Fig. 3. Scattering intensities per unit mass (I/M) for both (a) flaming and (b) non-flaming fires at 635 and (c) flaming and (d) non-flaming fires at $532 \mathrm{~nm}$ vs. the modulus of the scattering vector, $q$.

Also shown in Fig. 2 are the intensities measured at the four forward angles for diesel particulate matter (DPM) that, although obtained during a different study, were acquired with the same six-angle scattering module using a $\mathrm{HeNe}$ laser with an output wavelength of $632.8 \mathrm{~nm}$ [17]. What is immediately evident from the DPM data is the rapid decrease in intensity as the forward angle increases. For instance, at $30^{\circ}$ the intensity is only one-fourth of the intensity for the aerosol from a flaming fire, and one-sixth the intensity from that of the non-flaming fire. These differences are quite significant, most likely due to the smaller size of DPM and possibly due to greater carbon content that reduces the scattering intensity. Perhaps of greater interest would be the utilization of these differences to discriminate aerosols from different sources, either to improve smoke detector performance or to assist in the measurement and characterization of atmospheric aerosols. Figure 3 represents the scattering intensities of the fractal aggregates per unit mass concentration 
obtained at the two wavelengths, $635 \mathrm{~nm}$ and $532 \mathrm{~nm}$, in order to ascertain what, if any, differences exist in terms of the scattering sensitivity at different wavelengths. Because of differences in the power levels, beam diameters and photodiode sensitivities at the two wavelengths, the sensitivity data at one wavelength cannot be compared directly to the sensitivity at the other wavelength. However, with the exception of the data for aerosols from polypropylene, for which no data were acquired at $635 \mathrm{~nm}$, the relative sensitivities appear to vary in a similar manner at each of the wavelengths used. For both the flaming and non-flaming aerosols, the intensities per unit mass concentration are significantly higher (typically, by a factor of 2 to 4 ) at the lower angles of $15^{\circ}$ and $22^{1} 2^{\circ}$, indicating that the use of forward angles in this range have higher sensitivity for detection and measurement of lower aerosol mass concentrations.

Representative SEM/TEM photographs from samples collected from flaming and non-flaming fires for combustible materials used in the present study are shown in Fig. 4. It is evident from these photographs that smoke produced from open flaming fires are fractal aggregates with relatively small primary particles and elongated, chain-like morphologies (smaller $D_{f}$ ), while those produced from non-flaming fires have larger primary particles and clumped or more densely packed morphologies (larger $D_{f}$ ).

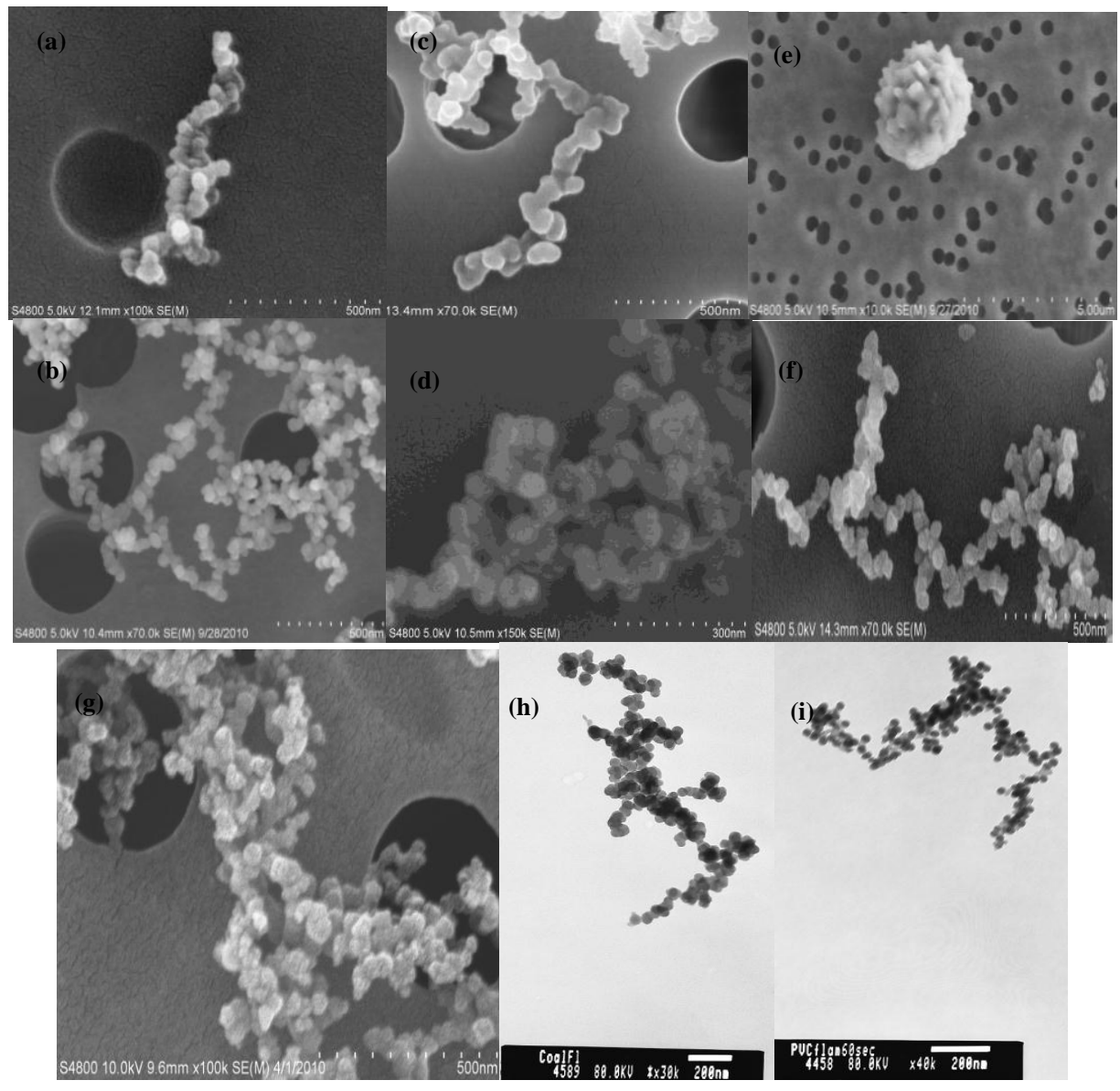

Fig. 4. SEM images of aggregate particles generated from: (a) non-flaming and (b) flaming Douglas-fir wood; (c) non-flaming and (d) flaming SBR rubber; (e) non-flaming and (f) flaming polypropylene; (g) flaming coal; and TEM images of (h) flaming coal and (i) flaming PVC rubber.

As discussed in the theory section, light extinction data were obtained using both broadband visible light and monochromatic electromagnetic radiation at $\lambda=532 \mathrm{~nm}$, and total scattering coefficient was measured using an integrating nephelometer operating at $\lambda=520 \mathrm{~nm}$. From these data, the mass absorption, mass extinction and mass scattering coefficients were calculated and are shown in Table 1 below. The obtained data are remarkable for several reasons. First, the mass extinction coefficients for aerosols from the flaming fires are significantly higher than values reported in the literature, while the mass scattering coefficients are relatively constant, resulting in very high mass absorption coefficients. The average mass absorption 
coefficient for the flaming fire aerosols is $13.7 \mathrm{~m}^{2} / \mathrm{g}$-almost twice the average value of $7.5 \mathrm{~m}^{2} / \mathrm{g}$ reported by Bond and Bergstrom, even though the average albedo measured here is 0.28 , compared to their average reported value of 0.25 [5]. While the mass extinction coefficients for aerosols from the non-flaming fires are reasonable (except for the very high value found for polypropylene-more than twice the average of the other three non-flaming combustion sources), the observation that the mass scattering coefficients are so large, with a resulting albedo that averages 0.87 , is remarkable considering that previously reported albedos are less than half this value. The high value for the polypropylene may be due to the extreme clumping of the primary particles, producing an almost spherical aggregate as can be seen in Fig. 4e, and for which classical Mie scattering theory may be more applicable in describing the optical properties.

Table 1. Scattering, extinction and absorption coefficients for aerosols for the different combustion sources.

\begin{tabular}{|l|c|c|c|c|c|c|}
\hline Flaming & $\boldsymbol{\sigma}_{\text {ext }}(\mathbf{5 3 2} \mathbf{~ n m})$ & $\boldsymbol{\sigma}_{\text {sca }}(\mathbf{5 2 0} \mathbf{~ n m})$ & $\boldsymbol{\sigma}_{\text {abs }}(\mathbf{5 3 2} \mathbf{~ n m})$ & $\boldsymbol{\sigma}_{\text {ext }}($ visible $)$ & Albedo & OD \\
\hline Douglas-fir & 16.7 & 3.3 & 13.4 & 13.7 & 0.20 & 0.073 \\
\hline Pgh coal & 12.9 & 4.5 & 8.4 & 11.2 & 0.35 & 0.058 \\
\hline SBR rubber & 16.1 & 5.5 & 10.6 & 11.1 & 0.34 & 0.070 \\
\hline Polypropylene & 26.6 & 7.3 & 19.3 & 17.8 & 0.27 & 0.092 \\
\hline No. 2 diesel fuel & 22.1 & 5.5 & 16.6 & 20.2 & 0.25 & 0.096 \\
\hline Non-flaming & & & & & & \\
\hline Douglas-fir & 7.6 & 6.6 & 1.0 & 4.3 & 0.87 & 0.033 \\
\hline Pgh coal & 7.0 & 6.2 & 0.8 & 5.2 & 0.88 & 0.031 \\
\hline SBR rubber & 8.3 & 7.2 & 1.1 & 4.5 & 0.87 & 0.036 \\
\hline Polypropylene & 15.7 & 14.1 & 1.6 & 10.5 & 0.90 & 0.068 \\
\hline
\end{tabular}

These results can be used to estimate obscuration levels from typical fires that can impact a person's ability to safely egress during an actual fire situation. The last column of Table 1 is an estimate of the optical density assuming an aerosol mass concentration of $10 \mathrm{mg} / \mathrm{m}^{3}$. Using these values over a distance of $3.05 \mathrm{~m}$, the average obscuration (defined as $1-I / I_{0}$ ) resulting from aerosols from flaming fires is over $42 \%$, and $25 \%$ for aerosols from non-flaming fires. In a study by Jin [27], an optical density roughly equal to $0.22 \mathrm{~m}^{-1}$ (obscuration of $79 \%$ over a $3.05 \mathrm{~m}$ path) was the critical value that severely limited a person's ability to safely evacuate from a fire situation - even when that person was familiar with the escape route.

\section{Prototype Smoke Sensor Data}

As mentioned previously, the response of a combination ionization and optical scattering prototype smoke

Table 2. Characteristics of fractal aggregates measured and calculated from these studies.

\begin{tabular}{|c|c|c|c|c|c|c|}
\hline Flaming & Douglas-fir & Pgh coal & $\begin{array}{c}\text { SBR } \\
\text { rubber }\end{array}$ & Polypropylene & $\begin{array}{c}\text { No. 2 diesel } \\
\text { fuel }\end{array}$ & Average \\
\hline$R_{g}(\mathrm{~nm})$ & $2.70 \times 10^{2}$ & $3.25 \times 10^{2}$ & $2.52 \times 10^{2}$ & $2.91 \times 10^{2}$ & $2.90 \times 10^{2}$ & $2.86 \times 10^{2}$ \\
\hline$d_{g}(\mathrm{~nm})$ & $2.90 \times 10^{2}$ & $2.23 \times 10^{2}$ & $2.30 \times 10^{2}$ & $1.82 \times 10^{2}$ & $2.20 \times 10^{2}$ & $2.29 \times 10^{2}$ \\
\hline$N\left(\mathrm{p} / \mathrm{cm}^{3}\right)$ & $1.68 \times 10^{6}$ & $1.87 \times 10^{6}$ & $2.56 \times 10^{6}$ & $2.60 \times 10^{6}$ & $3.17 \times 10^{6}$ & $2.38 \times 10^{6}$ \\
\hline$M_{a}(\mathrm{~g})$ & $1.57 \times 10^{-14}$ & $8.75 \times 10^{-15}$ & $8.94 \times 10^{-15}$ & $1.08 \times 10^{-14}$ & $5.51 \times 10^{-15}$ & $9.96 \times 10^{-15}$ \\
\hline$d_{p}(\mathrm{~nm})$ & $4.10 \times 10^{1}$ & $2.26 \times 10^{1}$ & $3.61 \times 10^{1}$ & $2.62 \times 10^{1}$ & $1.21 \times 10^{1}$ & $2.76 \times 10^{1}$ \\
\hline$n_{p}$ & $4.52 \times 10^{3}$ & $1.54 \times 10^{3}$ & $7.29 \times 10^{2}$ & $1.60 \times 10^{4}$ & $2.38 \times 10^{3}$ & $5.04 \times 10^{3}$ \\
\hline$D_{f}$ & 2.34 & 1.77 & 1.73 & 1.67 & 1.69 & 1.84 \\
\hline$M\left(\mathrm{mg} / \mathrm{m}^{3}\right)$ & $1.91 \times 10^{1}$ & $1.55 \times 10^{1}$ & $1.94 \times 10^{1}$ & $2.00 \times 10^{1}$ & $1.68 \times 10^{1}$ & $1.82 \times 10^{1}$ \\
\hline Non-flaming & & & & & \\
\hline$R_{g}(\mathrm{~nm})$ & $2.37 \times 10^{2}$ & $2.27 \times 10^{2}$ & $2.37 \times 10^{2}$ & $2.06 \times 10^{2}$ & & $2.27 \times 10^{2}$ \\
\hline$d_{g}(\mathrm{~nm})$ & $3.49 \times 10^{2}$ & $4.00 \times 10^{2}$ & $3.83 \times 10^{2}$ & $3.77 \times 10^{2}$ & & $3.77 \times 10^{2}$ \\
\hline$N\left(\mathrm{p} / \mathrm{cm}^{3}\right)$ & $7.81 \times 10^{5}$ & $6.48 \times 10^{5}$ & $6.08 \times 10^{5}$ & $2.88 \times 10^{5}$ & & $5.81 \times 10^{5}$ \\
\hline$M_{a}(\mathrm{~g})$ & $2.91 \times 10^{-14}$ & $3.45 \times 10^{-14}$ & $4.04 \times 10^{-14}$ & $1.20 \times 10^{-13}$ & & $5.61 \times 10^{-14}$ \\
\hline$d_{p}(\mathrm{~nm})$ & $5.97 \times 10^{1}$ & $8.22 \times 10^{1}$ & $8.06 \times 10^{1}$ & $3.69 \times 10^{2}$ & & $1.48 \times 10^{2}$ \\
\hline$n_{p}$ & $3.44 \times 10^{2}$ & $1.44 \times 10^{2}$ & $4.24 \times 10^{2}$ & $3.08 \times 10^{1}$ & & $2.35 \times 10^{2}$ \\
\hline$D_{f}$ & 2.22 & 2.30 & 2.19 & 2.34 & & 2.26 \\
\hline$M\left(\mathrm{mg} / \mathrm{m}^{3}\right)$ & $2.22 \times 10^{1}$ & $1.88 \times 10^{1}$ & $2.27 \times 10^{1}$ & $1.98 \times 10^{1}$ & & $2.09 \times 10^{1}$ \\
\hline
\end{tabular}


sensor was also obtained during these experiments and the data analyzed in two different manners. First, as discussed above, data from the ionization chamber component of the sensor, along with parameters from the optical scattering data, were used to calculate properties of the fractal aggregates. The results of these computations, including the $R_{g}$, and $D_{f}$, from the optical data, are shown in Table 2 . The Table 2 data provide a unique and complete description of the physical properties of the differing fractal aggregates that has heretofore not been available in the literature. Analysis of this table shows several distinctive trends relative to the aerosols from flaming versus non-flaming fires. First, aerosols from flaming fires have larger values of $R_{g}$, but smaller values of primary particle diameter. The $D_{f}$ of aerosols from flaming fires have an average value of 1.80 , while for those from non-flaming fires the average is 2.26 , with both values consistent with data from the literature [6]. Flaming fires produce fractal aggregates with small primary particles but large numbers of primary particles per aggregate, with the result that the average mass of a fractal aggregate is more than 3 times lower than the corresponding average mass of FAs from non-flaming fires. It is also interesting to note that the average number mean diameter calculated for flaming FAs is $30 \%$ less than the average for non-flaming FAs, while the average $R_{g}$ for flaming FAs is $30 \%$ higher.The data for the prototype sensor are also analyzed in terms of its responses per unit mass concentration as functions of the dimensionless ratios of ionization signal voltage, $C E V$, to optical scattering voltages at both $15^{\circ}$ and $30^{\circ}, V_{15}$ and $V_{30}$, respectively. The average resulting ratios for the different combustion sources and two modes of combustion are shown in Table 3. From the data, it is readily apparent that ionization-type smoke detectors are more responsive to aerosols from flaming fires, while photoelectric-types are more responsive to aerosols from non-flaming fires. Further, the data actually quantify these observations for a wide range of combustible materials. For instance, the average sensitivity of the ionization chamber, $C E V / M$, to aerosols from flaming fires is 0.0586 , while for non-flaming aerosols the value is 0.0225 -more than a factor of two lower. Similarly, for the $15^{\circ}$ sensitivity, $V_{I 5} / M$, the average value is 0.0280 for flaming fire aerosols but increases dramatically to a value of 0.0393 for non-flaming aerosols.

Perhaps of even greater significance is the fact that the sensitivities are found to vary with the signal ratios - a dimensionless and easily measured quantity. This result is shown in Fig. 5 where the ionization chamber sensitivity, $C E V / M$, and $30^{\circ}$ angular scattering sensitivity, $V_{30} / M$, are plotted as a function of the ratio, $C E V / V_{30}$.

Table 3. Ionization chamber sensitivities, optical scattering sensitivities and signal ratios for the various combustion sources and two modes of combustion.

\begin{tabular}{|l|c|c|c|c|c|}
\hline Flaming & $\mathbf{V}_{\mathbf{1 5}} / \mathbf{M}$ & $\mathbf{V}_{\mathbf{3 0}} / \mathbf{M}$ & $\mathbf{C E V} / \mathbf{M}$ & $\mathbf{C E V} / \mathbf{1 5}$ & $\mathbf{C E V} / 30$ \\
\hline Douglas-fir & 0.0223 & 0.0111 & 0.0436 & 1.984 & 3.905 \\
\hline Pgh coal & 0.0311 & 0.0124 & 0.0601 & 2.001 & 5.001 \\
\hline SBR rubber & 0.0270 & 0.0106 & 0.0660 & 2.807 & 6.904 \\
\hline Polypropylene & 0.0263 & 0.0100 & 0.0795 & 3.193 & 13.698 \\
\hline No. 2 diesel fuel & 0.0337 & 0.0162 & 0.0713 & 2.117 & 4.424 \\
\hline Average & 0.0281 & 0.0121 & 0.0641 & 2.420 & 6.786 \\
\hline Non-flaming & & & & & \\
\hline Douglas-fir & 0.0292 & 0.0129 & 0.0217 & 0.740 & 1.683 \\
\hline Pgh coal & 0.0342 & 0.0143 & 0.0255 & 0.798 & 1.698 \\
\hline SBR rubber & 0.0385 & 0.0146 & 0.0192 & 0.590 & 1.554 \\
\hline Polypropylene & 0.0668 & 0.0196 & 0.0152 & 0.229 & 0.760 \\
\hline Average & 0.0422 & 0.0154 & 0.0204 & 0.589 & 1.424 \\
\hline
\end{tabular}

The results indicate that it is possible to utilize the simple ratio to define regions of sensitivity and regions of discrimination that can aid in the fire detection process as well as discriminate against other non-fire related sources. Application of the sensor as a smoke detector, for instance, in areas that may contain significant levels of diesel exhaust particles has been previously shown to work quite well, where DPM has a very high value of both $C E V / M$ and $C E V / 30$, allowing for very good discrimination capabilities $[18,19$, 28]. In addition, the use of the sensor to quantify either airborne aerosol mass concentrations or total aerosol surface area for health purposes is also being pursued. 


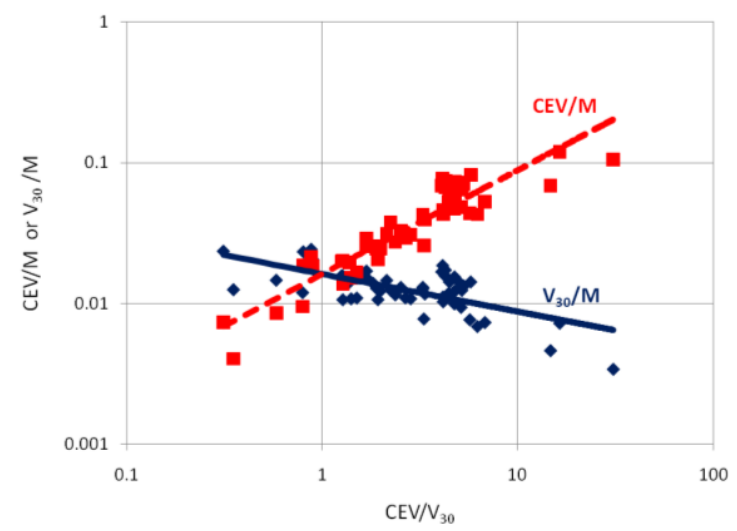

Fig. 5. Prototype detector sensitivities, CEV/M or $\mathrm{V}_{30} / \mathrm{M}$ vs CEV/ $\mathrm{V}_{30}$.

\section{Comparison of Results with Theory}

Clearly, the above data indicate that differences exist in the angular scattering signatures as well as for the extinction, scattering and absorption coefficients both as a function of the combustion source and whether the combustion is flaming or non-flaming. While it is beyond the scope of this paper to address in detail the individual source data, it is instructive to look at the detailed numerical calculations (discrete dipole approximation using DDSCAT) for the flaming and non-flaming cases in order to obtain some idea as to the aerosol properties most responsible for the observed differences. In these calculations, a standard fractal aggregate described in the literature consisting of 74 primary particles [24] with a nominal $D_{f}$ of 1.70 was used, and higher $D_{f}$ values were simulated by allowing the spheres to overlap in a manner similar to that reported by $\mathrm{Oh}$ and Sorensen [25]. For aerosols from the flaming fires, the average value of $D_{f}$ from Table 1 is 1.80 and for non-flaming fires 2.26, corresponding to overlaps of 1.4 and 3.5, respectively. For the flaming FAs, the volume equivalent diameter is $252 \mathrm{~nm}$ and for non-flaming FAs $342 \mathrm{~nm}$. From the data in Table 2, the average albedo at $\lambda=532 \mathrm{~nm}$ for flaming FAs is 0.313 and for non-flaming FAs 0.880 - significantly greater than values found in the literature [5]. The higher value for the non-flaming FAs indicates that these particles absorb very little, indicative of a refractive index with a low imaginary component, while for the flaming FAs just the opposite is true.
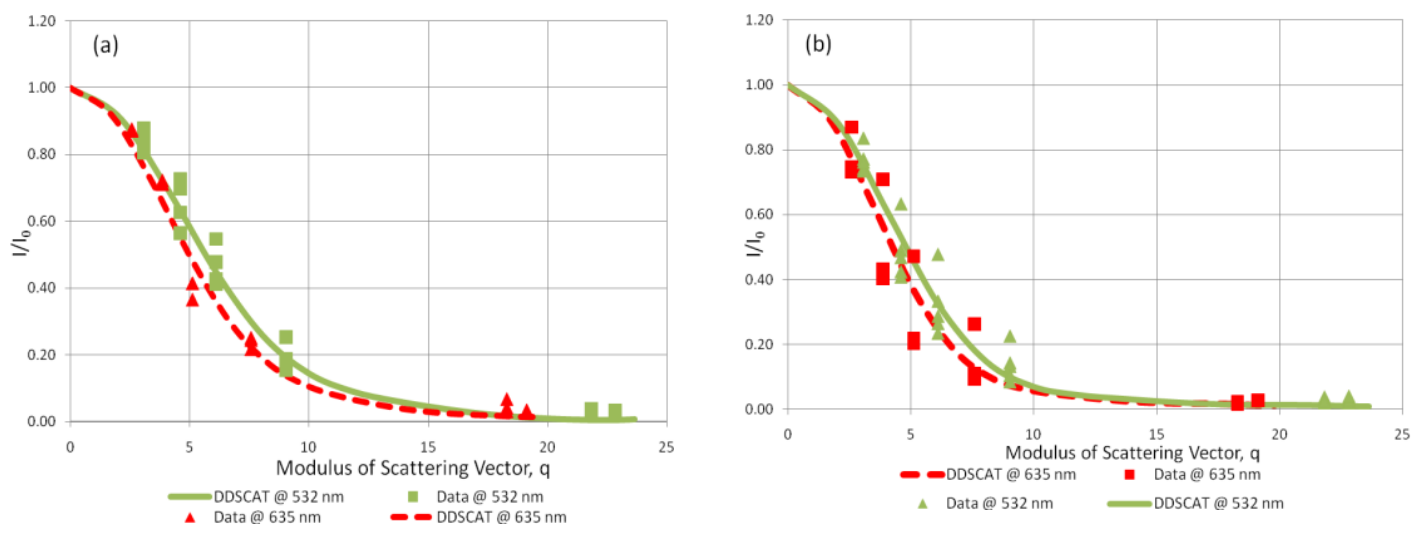

Fig. 6. Comparison of DDSCAT vs. measured scattering for (a) flaming and (b) non-flaming combustion.

Figure 6 shows the results of these computations for non-flaming FAs with a refractive index of $m=1.692$ $+0.0375 \mathrm{i}$, and flaming FAs with a refractive index of $m=1.692+1.25 \mathrm{i}$, where the corresponding numerically calculated albedos are 0.884 and 0.334 , respectively, in very good agreement with the measured values. For these numerical results, a volume equivalent radius of $212 \mathrm{~nm}$ was used for the nonflaming FAs and $190 \mathrm{~nm}$ for the flaming FAs. Although these values are somewhat higher than the values calculated using the data from Table 1, the qualitative agreement is quite good. For the numerically calculated curves of Fig. 6, the approximate fractal dimensions yielding the best agreement with the data are 1.79 for the flaming FAs and 2.16 for the non-flaming FAs, in excellent agreement with the 
experimental values cited above. Computations were also made using the RDG approximation discussed above. For these simpler computations, the parameters used were the average values found in Table 2 and included $d_{p}, R_{g}, n_{p}, D_{f}$, and the volume equivalent diameter was defined by $d_{e q}=d_{p}\left(n_{p}\right)^{1 / 3}$ for aerosols from both the flaming and non-flaming fires. The results of the calculated angular distributions are shown in Fig. 7, where for the flaming case the assumed index of refraction is $m=1.692+1.25 \mathrm{i}$, and for the nonflaming case $m=1.692+0.125$ i The meaning of the results is self-evident in that the agreement of these calculations with the data is excellent and the agreement comparable to the more detailed DDSCAT computations.
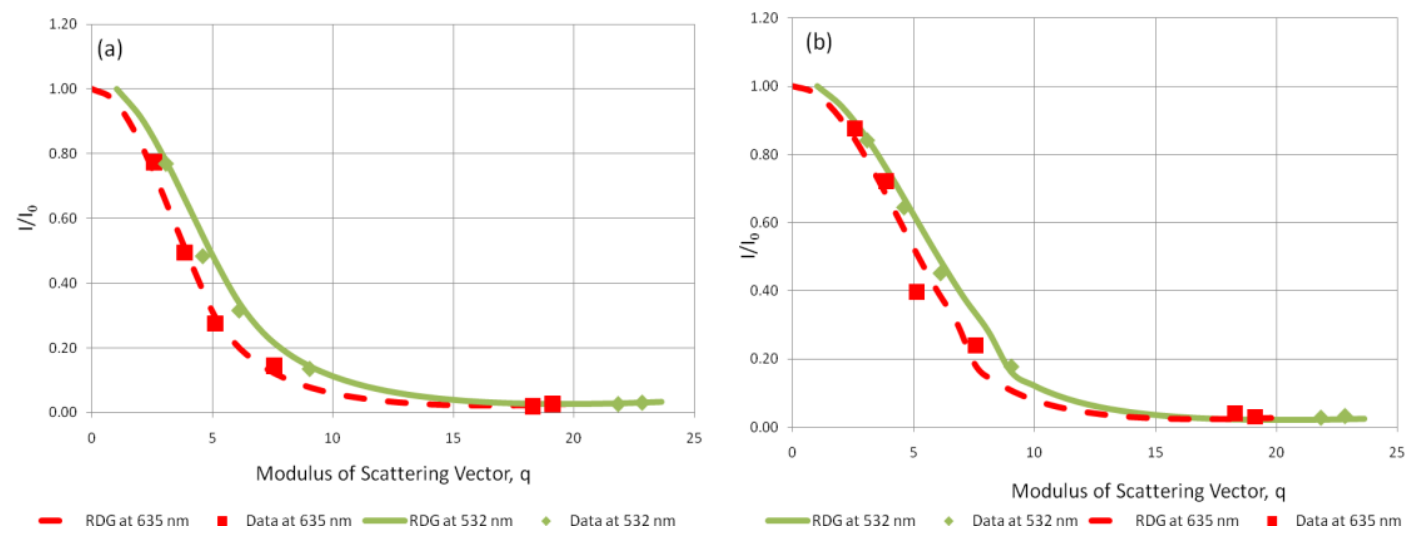

Fig. 7. Measured vs. calculated (RDG) scattering for (a) flaming; (b) non-flaming combustion aerosols.

Table 4. Measured coefficients versus those calculated using the RDG approximation.

\begin{tabular}{|c|c|c|}
\hline Average coefficient, $\mathbf{~}^{\mathbf{2}} / \mathbf{g}$ & Flaming data & Calculated from RDG \\
\hline$\sigma_{e x t}$ & $18.74(15.21)$ & 14.03 \\
\hline$\sigma_{s c a}$ & $5.36(4.41)$ & 4.19 \\
\hline$\sigma_{a b s}$ & $13.67(10.78)$ & 9.84 \\
\hline Albedo, $\sigma_{s c a} / \sigma_{e x t}$ & $0.28(0.30)$ & 0.30 \\
\hline$\sigma_{v i s}$ & 13.29 & \\
\hline & Non-flaming data & Calculated from RDG \\
\hline$\sigma_{e x t}$ & $9.37(7.63)$ & 8.67 \\
\hline$\sigma_{s c a}$ & $8.13(6.66)$ & 7.66 \\
\hline$\sigma_{a b s}$ & $1.24(0.97)$ & 1.01 \\
\hline Albedo, $\sigma_{s c a} / \sigma_{e x t}$ & 0.87 & 0.88 \\
\hline$\sigma_{v i s}$ & $6.41(4.67)$ & \\
\hline
\end{tabular}

In addition to the angular distributions, the mass extinction, scattering and absorption coefficients were also calculated and compared to the average experimental data. The results of this comparison are shown in Table 4. In this table, the average values in parentheses exclude the larger coefficients measured for the combustion sources of polypropylene and No. 2 diesel fuel, since the aerosol data from these two combustibles have a significant impact on the average. While there is not perfect agreement between the measured and calculated coefficients, it is believed that this agreement could be improved through minor changes in some of the parameters, including the index of refraction. But the fact that the calculated values are so close to those measured when using the parameter values obtained experimentally in Table 2 is remarkable, serving to reinforce the general applicability of the RDG approximation as an excellent tool for assessing the optical properties of fractal aggregates

\section{CONCLUSIONS}

The data and analysis presented above are a comprehensive summary of the optical and physical properties of fractal aggregates generated from a variety of combustible sources for both flaming and non-flaming fires. This summary represents a resource for future comparisons, more detailed analysis and development 
of improved smoke detectors as well as improved techniques for the monitoring, measurement and characterization of atmospheric carbonaceous aerosols. The data conclusively indicate that while all of the aerosols generated could be described as fractal aggregates, significant differences in their morphology and size exist both as a function of combustion source and combustion mode and that these differences result in different fractal properties that can subsequently be used to describe the fractal aggregates in analytical terms. While comparisons of the detailed theory using DDA and the simpler RDG approximation with the experimental data were more qualitative than quantitative, the results of the comparisons were quite excellent in the agreement between theory and experiment, providing a basis for expanding and refining the theoretical models, and, in particular, the RDG approximation as it applies to the fractal aggregates from combustion sources. Differences in radiative properties are most pronounced, in general, when the aerosols from flaming fires are compared to those from non-flaming fires, in that a large imaginary component of the index of refraction, $k_{e}$, indicative of very high carbon content, is needed in order to describe their optical properties theoretically; while for aerosols from non-flaming fires, a much smaller $k_{e}$ value, indicative of much lower carbon content, is necessary. Finally, the data quantify the response of an ionization chamber and optical scattering at discrete angles, thus indicating potential avenues for the design and improvement not only of smoke detectors but also of atmospheric aerosol monitors.

\section{ACKNOWLEDGEMENTS}

The authors would like to acknowledge Diane Schwegler-Berry at the National Institute for Occupational Safety and Health (NIOSH), Morgantown, WV, for her help in SEM/TEM imaging.

\section{REFERENCES}

[1] Dobbins, R.A., Mulholland, G.W., and Bryner, N.P., (1994) Comparison of a Fractal Smoke Optics Model with Light Extinction Measurements, Atmospheric Environment. 28(5): 889-897, http://dx.doi.org/10.1016/1352-2310(94)90247-X

[2] Singham, S.B. and Bohren, C.F., (1993) Scattering of Unpolarized and Polarized-Light by Particle Aggregates of Different Size and Fractal Dimension, Langmuir. 9(5): 1431-1435, http://dx.doi.org/10.1021/la00029a044

[3] Ayranci, I., Vaillon, R., Selcuk, N., Andre, F., and Escudie, D., (2007) Determination of soot temperature, volume fraction and refractive index from flame emission spectrometry, Journal of Quantitative Spectroscopy \& Radiative Transfer. 104(2): 266-276, http://dx.doi.org/10.1016/j.jqsrt.2006.07.013

[4] Bonczyk, P.A. and Hall, R.J., (1991) Fractal Properties of Soot Agglomerates, Langmuir. 7(6): 1274-1280, http://dx.doi.org/10.1021/la00054a042

[5] Bond, T.C. and Bergstrom, R.W., (2006) Light absorption by carbonaceous particles: An investigative review, Aerosol Science and Technology. 40(1): 27-67, http://dx.doi.org/10.1080/02786820500421521

[6] Brasil, A.M., Farias, T.L., and Carvalho, M.G., (2000) Evaluation of the fractal properties of cluster-cluster aggregates, Aerosol Science and Technology. 33(5): 440-454, http://dx.doi.org/10.1080/02786820050204682

[7] Chylek, P., Ramaswamy, V., Cheng, R., and Pinnick, R.G., (1981) Optical-Properties and Mass Concentration of Carbonaceous Smokes, Applied Optics. 20(17): 2980-2985, http://dx.doi.org/10.1364/AO.20.002980

[8] Fabian, T.Z. and Gandhi, P.D., (2007) Smoke characterization project, Final Report, Underwriters Laborotories, Inc.

[9] Gwaze, P., Schmid, O., Annegarn, H.J., Andreae, M.O., Huth, J., and Helas, G., (2006) Comparison of three methods of fractal analysis applied to soot aggregates from wood combustion, Journal of Aerosol Science. 37(7): 820-838, http://dx.doi.org/10.1016/j.jaerosci.2005.06.007

[10] Collinge, M.J. and Draine, B.T., (2004) Discrete-dipole approximation with polarizabilities that account for both finite wavelength and target geometry, Journal of the Optical Society of America 
a-Optics Image Science and Vision. 21(10): 2023-2028, http://dx.doi.org/10.1364/JOSAA.21.002023

[11] Draine, B.T., (1988) The Discrete-Dipole Approximation and its Application to Interstellar Graphite Grains, Astrophysical Journal. 333(2): 848-872, http://dx.doi.org/10.1086/166795

[12] Draine, B.T., (2003) Scattering by interstellar dust grains. I. Optical and ultraviolet, Astrophysical Journal. 598(2): 1017-1025, http://dx.doi.org/10.1086/379123

[13] Draine, B.T., (2003) Scattering by interstellar dust grains. II. X-rays, Astrophysical Journal. 598(2): 1026-1037, http://dx.doi.org/10.1364/JOSAA.11.001491

[14] Draine, B.T. and Flatau, P.J., (1994) Discrete-Dipole Approximation for Scattering Calculations, Journal of the Optical Society of America a-Optics Image Science and Vision. 11(4): 1491-1499, http://dx.doi.org/10.1364/JOSAA.11.001491

[15] Koylu, U.O. and Faeth, G.M., (1993) Radiative Properties of Flame-Generated Soot, Journal of Heat Transfer-Transactions of the ASME. 115(2): 409-417, http://dx.doi.org/10.1115/1.2910693

[16] Edwards, J.C., Morrow,G.S., (1995) Development of coal combustion sensitivity tests for smoke detectors, United States Department of Interior, Report of Investigation 9551. p. 1-12.

[17] Litton, C.D., (2002) The use of light scattering and ion chamber responses for the detection of fires in diesel contaminated atmospheres, Fire Safety Journal. 37(4): 409-425, http://dx.doi.org/10.1016/S0379-7112(01)00059-5

[18] Litton, C.D., (2009) Laboratory evaluation of smoke detectors for use in underground mines, Fire Safety Journal. 44(3): 387-393, http://dx.doi.org/10.1016/j.firesaf.2008.08.008

[19] Edwards, R., Smith, K.R., Kirby, B., Allen, T., Litton, C.D., and Hering, S., (2006) An inexpensive dual-chamber particle monitor: Laboratory characterization, Journal of the Air \& Waste Management Association. 56(6): 789-799.

[20] Farias, T.L., Carvalho, M.G., Koylu, U.O., and Faeth, G.M., (1995) Computational Evaluation of Approximate Rayleigh-Debye-Gans/Fractal-Aggregate Theory for the Absorption and Scattering Properties of Soot, Journal of Heat Transfer-Transactions of the ASME. 117(1): 152-159.

[21] Farias, T.L., Koylu, U.O., and Carvalho, M.G., (1996) Range of validity of the Rayleigh-DebyeGans theory for optics of fractal aggregates, Applied Optics. 35(33): 6560-6567, http://dx.doi.org/10.1364/AO.35.006560

[22] Sorensen, C.M., (2001) Light scattering by fractal aggregates: A review, Aerosol Science and Technology. 35(2): 648-687, http://dx.doi.org/10.1080/027868201316900007

[23] Litton, C.D., Smith, K.R., Edwards, R., and Allen, T., (2004) Combined optical and ionization measurement techniques for inexpensive characterization of micrometer and submicrometer aerosols, Aerosol Science and Technology. 38(11): 1054-1062, http://dx.doi.org/10.1080/027868290883333

[24] Ayranci, I., (2007) A nonintrusive diagnostics technique for flame soot based on near infrared emission spectroscopy, Thesis submitted to the Middle East Technical University. p. 1-188.

[25] Oh, C. and Sorensen, C.M., (1997) The effect of overlap between monomers on the determination of fractal cluster morphology, Journal of Colloid and Interface Science. 193(1): p. 17-25, http://dx.doi.org/10.1006/jcis.1997.5046

[26] Dobbins, R.A. and Megaridis, C.M., (1991) Absorption and Scattering of Light by Polydisperse Aggregates, Applied Optics. 30(33): 4747-4754, http://dx.doi.org/10.1364/AO.30.004747

[27] Jin, T., (1981) Studies of Emotional Instability in Smoke from Fires, Journal of Fire \& Flammability. 12(2): 130-142.

[28] Litton, C.D., (2002) Studies of the measurement of respirable coal dusts and diesel particulate matter, Measurement Science \& Technology. 13(3): 365-374, 